\title{
Formation of Arylboronates by a Cul-Catalyzed Coupling Reaction of Pinacolborane with Aryl lodides at Room Temperature
}

\author{
Wei Zhu ${ }^{1}$ and Dawei $\mathrm{Ma}^{2 *}$ \\ ${ }^{1}$ Department of Chemistry, Fudan University, Shanghai 200433,China ${ }^{2}$ State Key \\ Laboratory of Bioorganic and Natural Products Chemistry, Shanghai Institute of \\ Organic Chemistry, Chinese Academy of Sciences, 354 Fenglin Lu, Shanghai 200032, \\ China
}

Supporting information

Table of contents

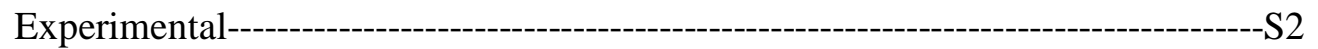

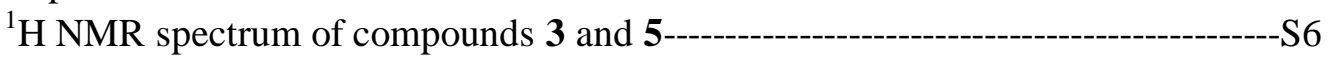




\section{Experimental}

\section{General Procedure for CuI-catalyzed cross-coupling reaction of}

4-iodoanisole 1a with pinacolborane 2: To a solution of 4-iodoanisole (1 mmol), copper salt $(0.1 \mathrm{mmol})$, base $(1.5 \mathrm{mmol})$ and additive $(0 \sim 0.2 \mathrm{mmol})$ in THF $(4 \mathrm{~mL})$ was added pinacolborane $(1.5 \mathrm{mmol})$ via syringe under argon atmosphere. The resultant mixture was stirred at appropriate temperature for $24 \mathrm{~h}$ or until the aryl iodide disappeared monitored by TLC. After the reaction was quenched by adding $5 \mathrm{~mL}$ of saturated $\mathrm{NH}_{4} \mathrm{Cl}$, the mixture was extracted with ethyl acetate. The combined organic layers were washed with brine and dried over $\mathrm{MgSO}_{4}$. The solution was concentrated under vacuo and the residue was chromatographed eluting with $1 / 10$ ethyl acetate/petroleum ether to afford aryl boronate.

\section{General Procedure for CuI-catalyzed cross-coupling reaction of aryl} iodides with pinacolborane: To a solution of of aryl iodide ( 2 mmol), copper salt $(0.2$ mmol) and sodium hydride $(3 \mathrm{mmol})$ in THF $(8 \mathrm{~mL})$ was added pinacolborane $(3$ mmol) via syringe under argon atmosphere. The resultant mixture was stirred at room until the aryl iodide disappeared monitored by TLC. After the reaction was quenched by adding $10 \mathrm{~mL}$ of saturated $\mathrm{NH}_{4} \mathrm{Cl}$, the mixture was extracted with ethyl acetate. The combined organic layers were washed with brine and dried over $\mathrm{MgSO}_{4}$. The solution was concentrated under vacuo and the residue was chromatographed eluting with $1 / 10$ ethyl acetate/petroleum ether to afford aryl boronates.

2-(4-Methoxy-phenyl)-4,4,5,5-tetramethyl-[1,3,2]dioxaborolane $\quad 3 a . \quad{ }^{1} \mathrm{H}$ 
NMR (300 MHz, $\left.\mathrm{CDCl}_{3}\right) \delta 7.76(\mathrm{~d}, J=8.7 \mathrm{~Hz}, 2 \mathrm{H}), 6.90(\mathrm{~d}, J=9.0 \mathrm{~Hz}, 2 \mathrm{H}), 3.83(\mathrm{~s}$, 3H), 1.33 (s, 12H); EI-MS m/z $234\left(\mathrm{M}^{+}\right)$, 219, 203, 191, 176, 161, 148, 134, 120, 104, $91,85,77,65,57,41$.

4,4,5,5-Tetramethyl-2-phenyl-[1,3,2] dioxaborolane 3b. ${ }^{1} \mathrm{H}$ NMR $(300 \mathrm{MHz}$, $\left.\mathrm{CDCl}_{3}\right) \delta 7.81(\mathrm{~d}, J=8.1 \mathrm{~Hz}, 2 \mathrm{H}), 7.45(\mathrm{~m}, 1 \mathrm{H}), 7.36(\mathrm{~m}, 2 \mathrm{H}), 1.35(\mathrm{~s}, 12 \mathrm{H})$; EI-MS $m / z 204\left(\mathbf{M}^{+}\right), 189,173,161,154,147,131,118,105,91,85,77,69,59,43$.

4-(4,4,5,5-Tetramethyl-[1,3,2] dioxaborolan-2-yl)phenol 3c. ${ }^{1} \mathrm{H}$ NMR (300 $\left.\mathrm{MHz}, \mathrm{CDCl}_{3}\right) \delta 7.70(\mathrm{~d}, J=8.7 \mathrm{~Hz}, 2 \mathrm{H}), 6.81(\mathrm{~d}, J=8.7 \mathrm{~Hz}, 2 \mathrm{H}), 5.76(\mathrm{~s}, 1 \mathrm{H}), 1.34(\mathrm{~s}$, 12H); EI-MS m/z $220\left(\mathrm{M}^{+}\right)$, 205, 189, 177, 163, 147, 134, 121, 103, 92, 85, 77, 66, 59, 41; HRMS found $m / z 220.1269\left(\mathrm{M}^{+}\right), \mathrm{C}_{12} \mathrm{H}_{17} \mathrm{BO}_{3}$ requires 220.1271 .

4,4,5,5-Tetramethyl-2-o-tolyl-[1,3,2] dioxaborolane 3d. ${ }^{1} \mathrm{H}$ NMR $(300 \mathrm{MHz}$, $\left.\mathrm{CDCl}_{3}\right) \delta 7.75(\mathrm{~m}, 1 \mathrm{H}), 7.30(\mathrm{~m}, 1 \mathrm{H}), 7.15(\mathrm{~m}, 2 \mathrm{H}), 2.54(\mathrm{~s}, 3 \mathrm{H}), 1.34$ (s, 12H); EI-MS $m / z 218\left(\mathbf{M}^{+}\right), 203,187,175,161,145,132,119,101,91,85,77,69,57,43$.

4,4,5,5-Tetramethyl-2-naphthalen-1-yl-[1,3,2]dioxaborolane 3e. ${ }^{1} \mathrm{H}$ NMR $\left(300 \mathrm{MHz}, \mathrm{CDCl}_{3}\right) \delta 8.78(\mathrm{~d}, J=8.4 \mathrm{~Hz}, 1 \mathrm{H}), 8.09(\mathrm{~d}, J=6.9 \mathrm{~Hz}, 1 \mathrm{H}), 7.95(\mathrm{~d}, J=7.8$ $\mathrm{Hz}, 1 \mathrm{H}), 7.85(\mathrm{~d}, J=8.1 \mathrm{~Hz}, 1 \mathrm{H}), 7.56-7.46(\mathrm{~m}, 3 \mathrm{H}), 1.44(\mathrm{~s}, 12 \mathrm{H})$; EI-MS m/z 254 $\left(\mathrm{M}^{+}\right), 239,210,196,181,168,154,141,128,102,85,77,69,57,41$.

2-(3-Methoxyphenyl)-4,4,5,5-tetramethyl-[1,3,2]dioxaborolane $\quad$ 3f. $\quad{ }^{1} \mathrm{H}$ NMR $\left(300 \mathrm{MHz}, \mathrm{CDCl}_{3}\right) \delta$ 7.41-7.25 (m, 3H), $7.05(\mathrm{~m}, 1 \mathrm{H}), 3.83(\mathrm{~s}, 3 \mathrm{H}), 1.37(\mathrm{~s}$, 12H); EI-MS m/z $234\left(\mathrm{M}^{+}\right)$, 219, 203, 191, 175, 161, 148, 134, 104, 91, 85, 77, 65, 57, 41.

2-(4-Iodophenyl)-4,4,5,5-tetramethyl-[1,3,2]dioxaborolane $3 g .{ }^{1} \mathrm{H}$ NMR 
$\left(300 \mathrm{MHz}, \mathrm{CDCl}_{3}\right) \delta 7.72(\mathrm{~d}, J=8.7 \mathrm{~Hz}, 2 \mathrm{H}), 7.51(\mathrm{~d}, J=8.7 \mathrm{~Hz}, 2 \mathrm{H}), 1.34(\mathrm{~s}, 12 \mathrm{H})$;

EI-MS m/z $330\left(\mathrm{M}^{+}\right)$, 315, 299, 287, 273, 244, 230, 217, 203, 188, 173, 161, 145, 117 , 104, 94, 85, 77, 69, 59, 41. HRMS found $m / z 330.0287\left(\mathrm{M}^{+}\right), \mathrm{C}_{12} \mathrm{H}_{16} \mathrm{BIO}_{2}$ requires 330.0292 .

2-(4-Bromophenyl)-4,4,5,5-tetramethyl-[1,3,2]dioxaborolane $3 \mathrm{~h} .{ }^{1} \mathrm{H}$ NMR $\left(300 \mathrm{MHz}, \mathrm{CDCl}_{3}\right) \delta 7.59(\mathrm{~m}, 2 \mathrm{H}) 7.43(\mathrm{~m}, 2 \mathrm{H}), 1.27(\mathrm{~s}, 12 \mathrm{H}) ;$ EI-MS m/z $282\left(\mathrm{M}^{+}\right)$, $267,253,239,225,204,196,183,161,146,129,117,103,85,77,69,59,41$.

2-(2-Fluorophenyl)-4,4,5,5-tetramethyl-[1,3,2]dioxaborolane $3 \mathrm{i} .{ }^{1} \mathrm{H}$ NMR $\left(300 \mathrm{MHz}, \mathrm{CDCl}_{3}\right) \delta 7.74(\mathrm{~m}, 1 \mathrm{H}), 7.43(\mathrm{~m}, 1 \mathrm{H}), 7.14(\mathrm{~m}, 1 \mathrm{H}), 7.04(\mathrm{~m}, 1 \mathrm{H}), 1.36(\mathrm{~s}$, 12H); EI-MS m/z $222\left(\mathrm{M}^{+}\right)$, 207, 189, 179, 160, 153, 149, 131, 123, 118, 105, 91, 85, 77, 69, 59, 43, 41. HRMS found $m / z 222.1223\left(\mathrm{M}^{+}\right), \mathrm{C}_{12} \mathrm{H}_{16} \mathrm{BFO}_{2}$ requires 222.1228.

4,4,5,5-Tetramethyl-2-(3-trifluoromethyl-phenyl)-[1,3,2]dioxaborolane $3 \mathrm{j}$. ${ }^{1} \mathrm{H}$ NMR (300 MHz, $\left.\mathrm{CDCl}_{3}\right) \delta 7.99(\mathrm{~s}, 1 \mathrm{H}), 7.90(\mathrm{~d}, J=7.5 \mathrm{~Hz}, 1 \mathrm{H}), 7.62(\mathrm{~m}, 1 \mathrm{H})$, $7.41(\mathrm{t}, J=8.1 \mathrm{~Hz}, 1 \mathrm{H}), 1.28(\mathrm{~s}, 12 \mathrm{H})$; EI-MS $m / z 272\left(\mathrm{M}^{+}\right), 257,241,215,186,173$, $153,145,117,85,77,69,58,43$. HRMS found $m / z 272.1187\left(\mathrm{M}^{+}\right), \mathrm{C}_{13} \mathrm{H}_{16} \mathrm{BF}_{3} \mathrm{O}_{2}$ requires 272.1196 .

4,4,5,5-Tetramethyl-2-thiophen-2-yl-[1,3,2]dioxaborolane $3 \mathrm{k} .{ }^{1} \mathrm{H} \quad \mathrm{NMR}$ $\left(300 \mathrm{MHz}, \mathrm{CDCl}_{3}\right) \delta 7.57(\mathrm{~m}, 2 \mathrm{H}), 7.12(\mathrm{~m}, 1 \mathrm{H}), 1.17(\mathrm{~s}, 12 \mathrm{H}) ;$ EI-MS m/z $330\left(\mathrm{M}^{+}\right)$, $315,299,287,273,257,244,231,215,202,188,173,158,144,131,117,101,85,79$, 69, 59, 43.

4,4,5,5-Tetramethyl-2-[4-(2-methyl-[1,3]dioxolan-2-yl)-phenyl]-[1,3,2]

dioxaborolane 3m. ${ }^{1} \mathrm{H}$ NMR $\left(300 \mathrm{MHz}, \mathrm{CDCl}_{3}\right) \delta 7.79(\mathrm{~d}, J=8.1 \mathrm{~Hz}, 2 \mathrm{H}), 7.49(\mathrm{~d}, J$ 
$=8.1 \mathrm{~Hz}, 2 \mathrm{H}), 4.06-4.01(\mathrm{~m}, 2 \mathrm{H}), 3.77-3.72(\mathrm{~m}, 2 \mathrm{H}), 1.65(\mathrm{~s}, 3 \mathrm{H}), 1.34(\mathrm{~s}, 12 \mathrm{H}) ;{ }^{13} \mathrm{C}$ NMR (75 MHz, $\left.\mathrm{CDCl}_{3}\right) \delta 145.92,134.37,124.24,108.40,83.40,64.03,27.14,24.47$;

EI-MS m/z $275\left(\mathrm{M}-\mathrm{CH}_{3}\right)^{+}, 259,231,217,191,175,159,147,131,119,104,87,77$, 57, 43; HRMS found $m / z 275.1455\left(\mathrm{M}-\mathrm{CH}_{3}\right)^{+}, \mathrm{C}_{16} \mathrm{H}_{23} \mathrm{BO}_{4}$ requires 290.1689, $\mathrm{C}_{15} \mathrm{H}_{21} \mathrm{BO}_{4}$ requires 275.1455 .

4-(4,4,5,5-Tetramethyl-[1,3,2] dioxaborolan-2-yl)-benzoic acid tert-butyl

ester 3n. ${ }^{1} \mathrm{H}$ NMR $\left(300 \mathrm{MHz}, \mathrm{CDCl}_{3}\right) \delta 7.96(\mathrm{~d}, J=8.1 \mathrm{~Hz}, 2 \mathrm{H}), 7.83(\mathrm{~d}, J=8.1 \mathrm{~Hz}$, 2H), $1.60(\mathrm{~s}, 9 \mathrm{H}), 1.36(\mathrm{~s}, 12 \mathrm{H}) ;{ }^{13} \mathrm{C} \mathrm{NMR}\left(75 \mathrm{MHz}, \mathrm{CDCl}_{3}\right) \delta 165.38,134.12,133.83$ 128.01, 83.70, 80.67, 27.79, 24.49; EI-MS m/z $304\left(\mathrm{M}^{+}\right), 289,249,233,218,205,189$, $173,162,149,131,118,104,85,77,57,41 ;$ HRMS found $m / z 304.1830\left(\mathrm{M}^{+}\right)$ $\mathrm{C}_{17} \mathrm{H}_{25} \mathrm{BO}_{4}$ requires 304.1846 .

\section{2-Dibenzylamino-3-[4-(4,4,5,5-tetramethyl-[1,3,2]dioxaborolan-2-yl)-}

phenyl]-propionic acid tert-butyl ester 5. $[\alpha]_{\mathrm{D}}{ }^{26}=-34.9\left(c \quad 1.5, \mathrm{CHCl}_{3}\right),{ }^{1} \mathrm{H} \mathrm{NMR}$ $\left(300 \mathrm{MHz}, \mathrm{CDCl}_{3}\right) \delta 7.66(\mathrm{~d}, J=7.8 \mathrm{~Hz}, 2 \mathrm{H}), 7.27-7.16(\mathrm{~m}, 10 \mathrm{H}), 7.03(\mathrm{~d}, J=8.4 \mathrm{~Hz}$ 2H), $3.94(\mathrm{~d}, J=14.1 \mathrm{~Hz}, 2 \mathrm{H}), 3.57(\mathrm{~d}, J=13.8 \mathrm{~Hz}, 2 \mathrm{H}), 3.52(\mathrm{~m}, 1 \mathrm{H}), 3.12-2.90(\mathrm{~m}$, 2H), $1.51(\mathrm{~s}, 9 \mathrm{H}), 1.36(\mathrm{~s}, 12 \mathrm{H}) ;{ }^{13} \mathrm{C} \mathrm{NMR}\left(75 \mathrm{MHz}, \mathrm{CDCl}_{3}\right) \delta 171.56,142.11,139.54$ $134.74,129.02,128.82,128.27,126.99,83.79,81.30,62.75,54.58,36.08,29.84$ 28.54, 25.00; ESI-MS m/z $528.3(\mathrm{M}+\mathrm{H})^{+}$; ESI-HRMS found $m / z 528.3278(\mathrm{M}+\mathrm{H})^{+}$, $\mathrm{C}_{33} \mathrm{H}_{43} \mathrm{BNO}_{4}$ requires 528.3279 . 

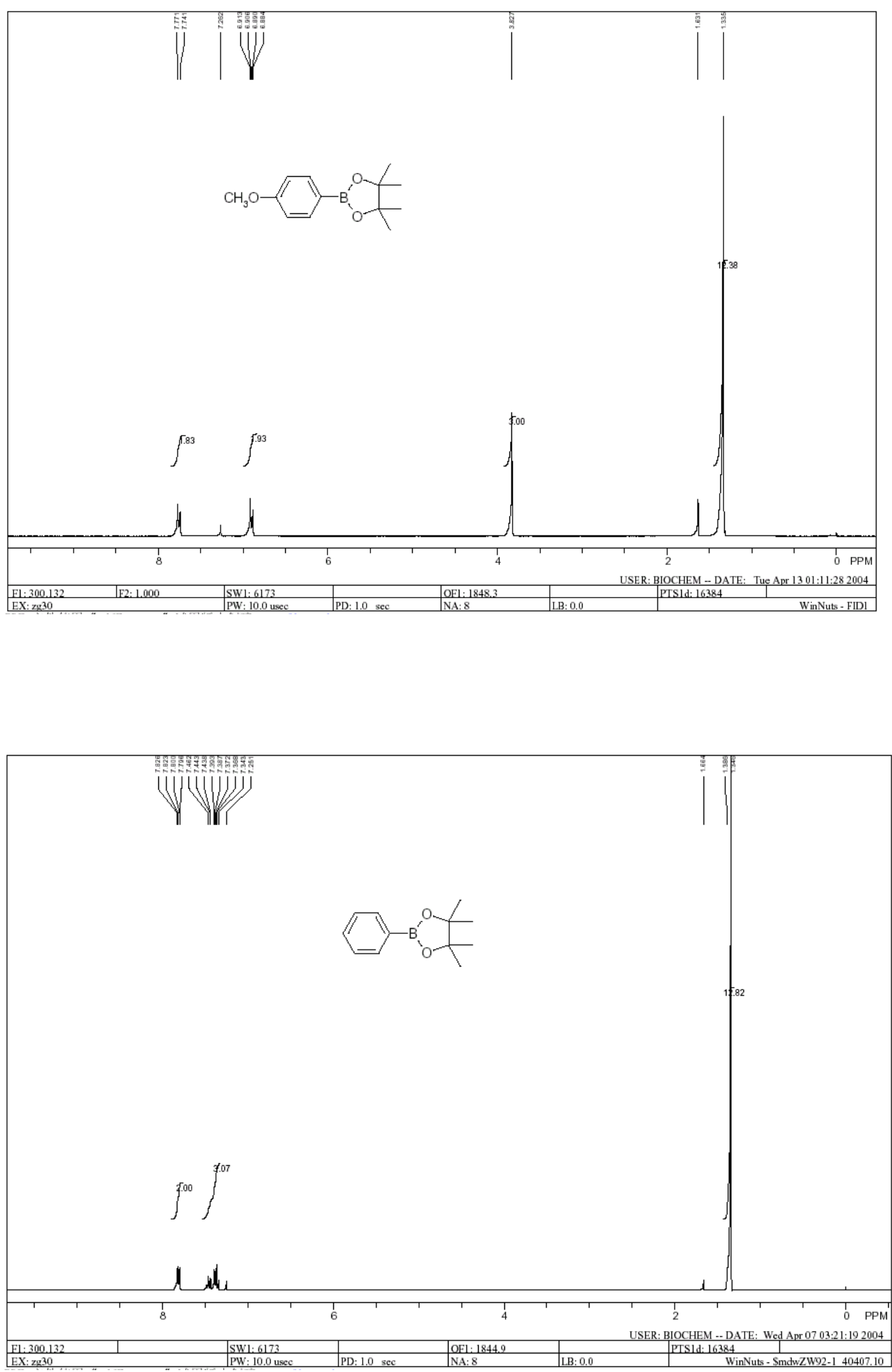

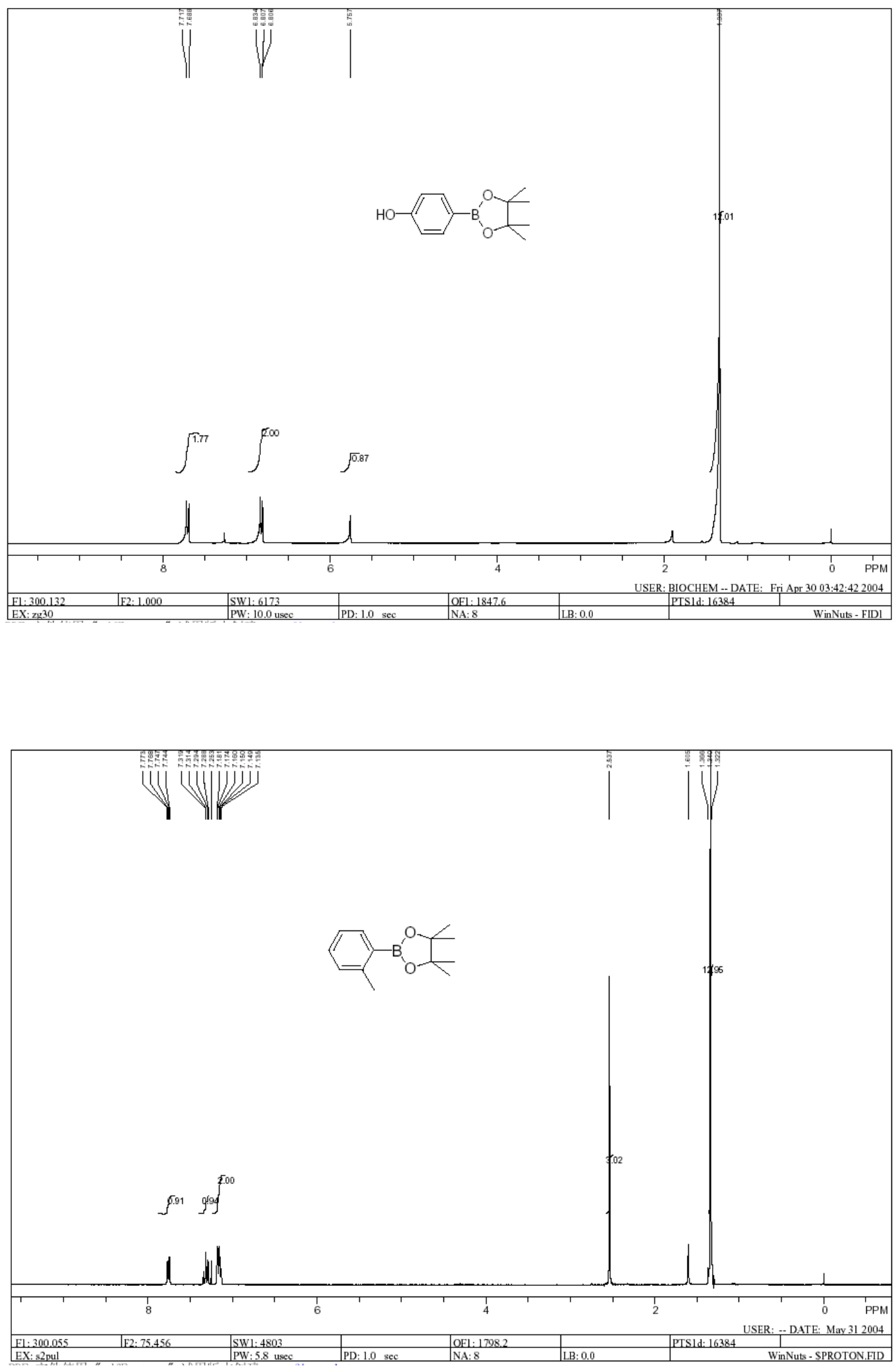

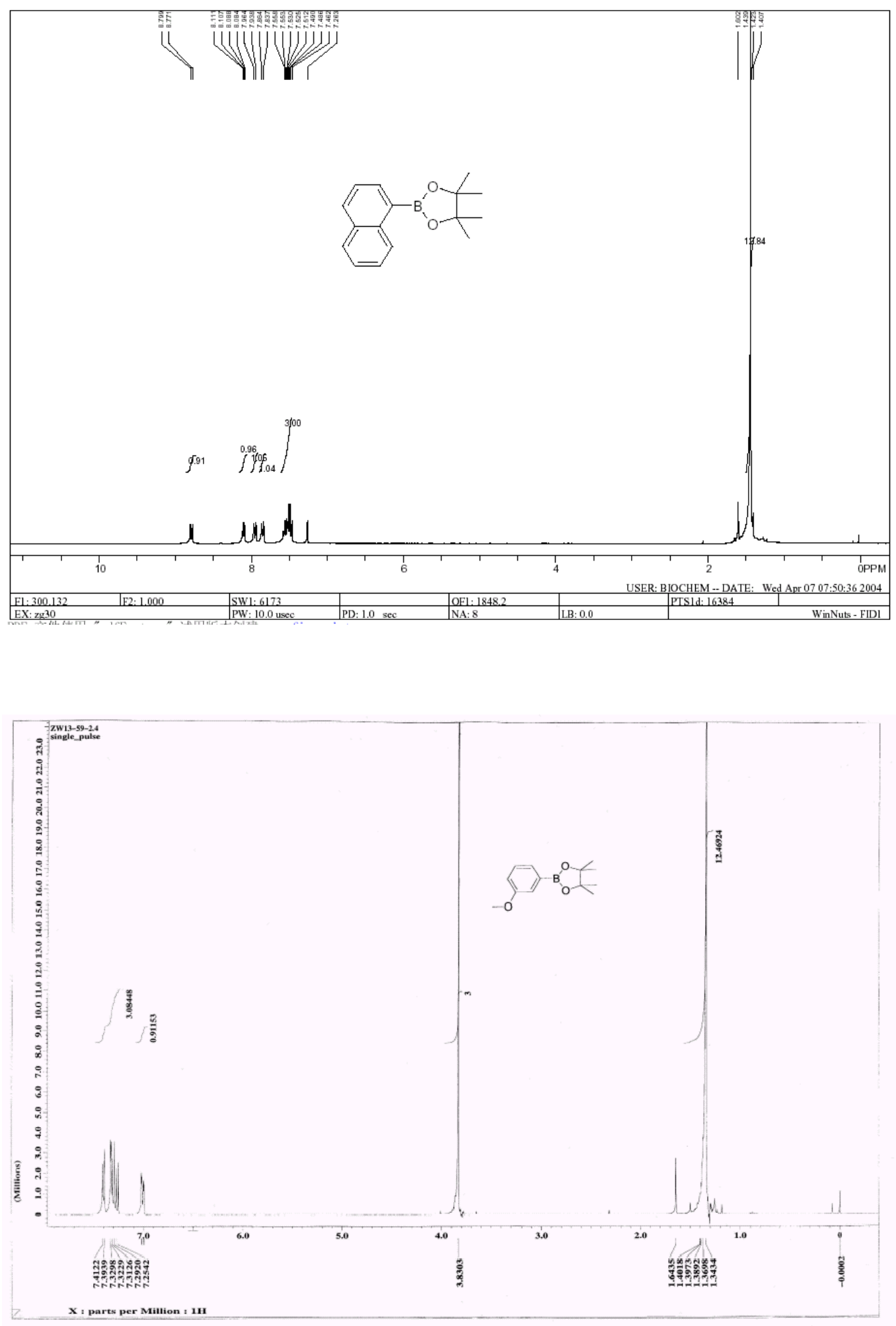

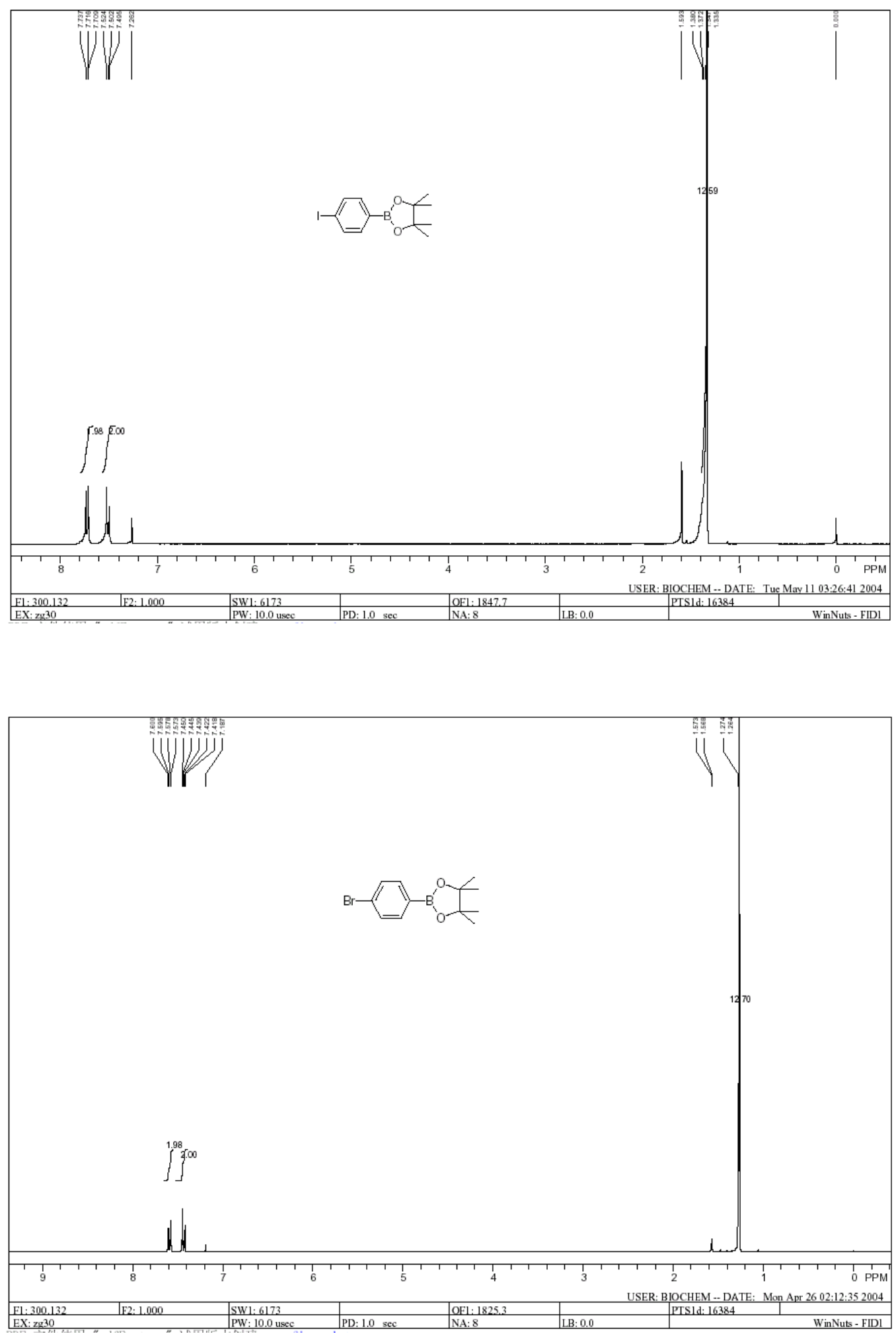

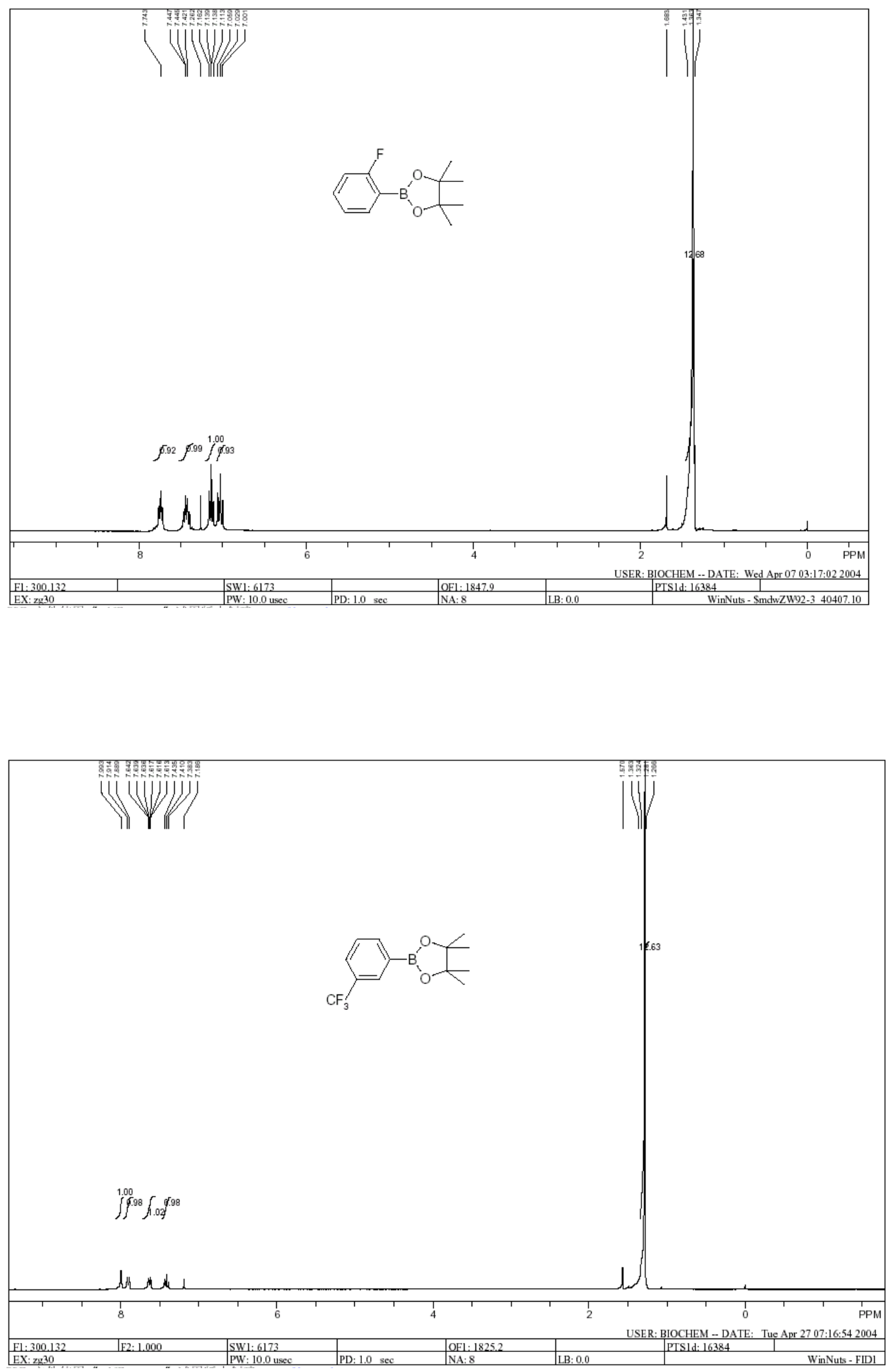

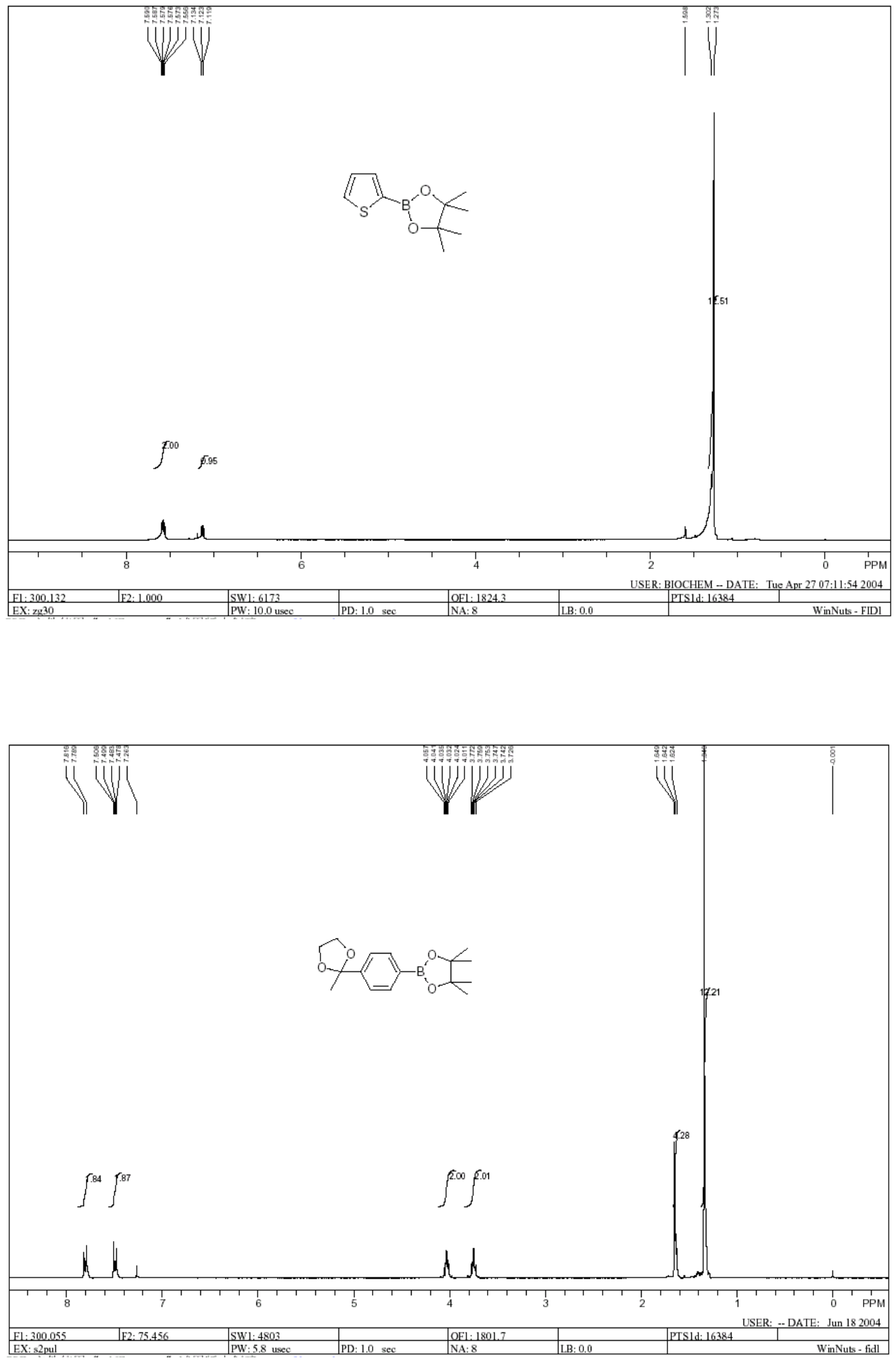

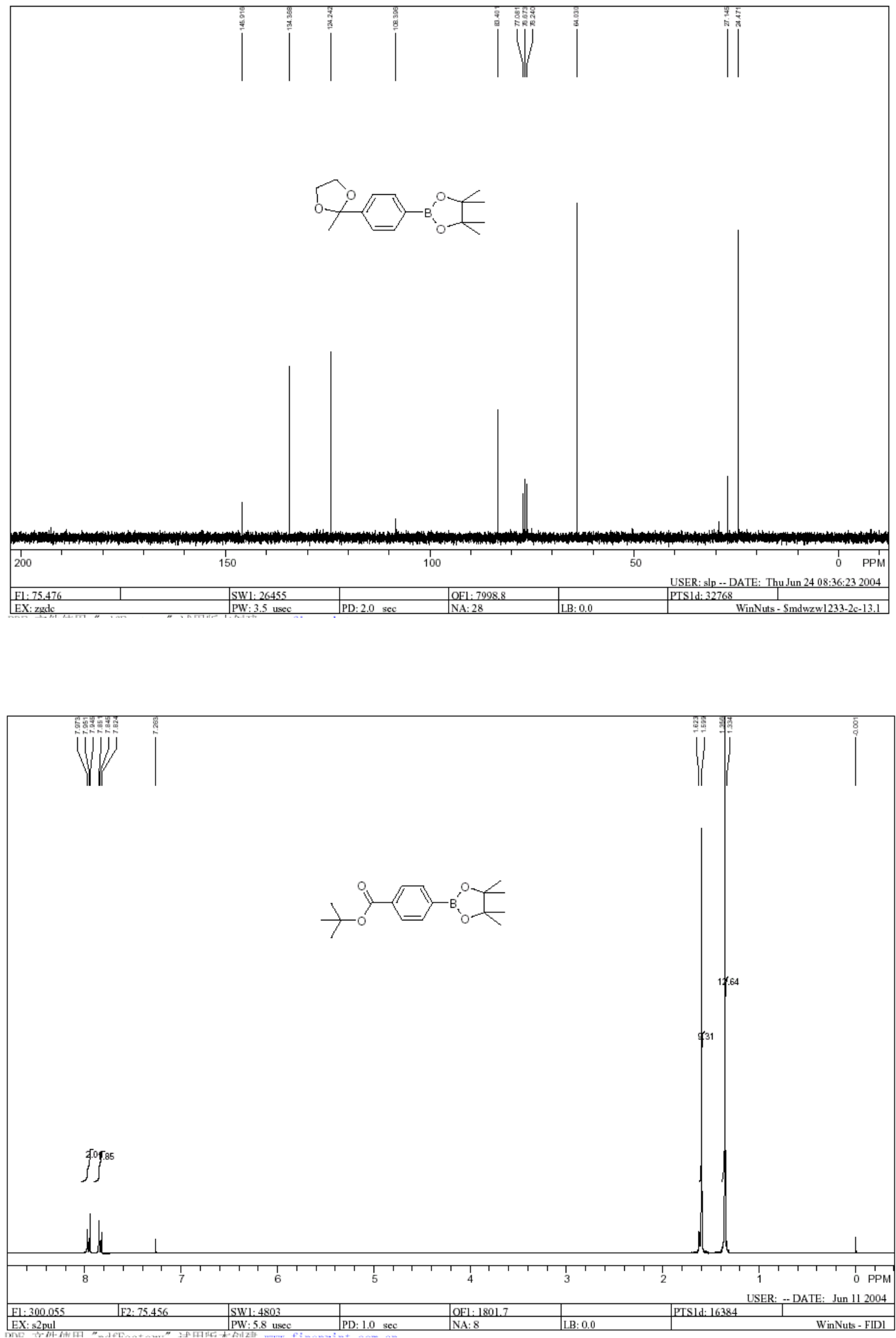

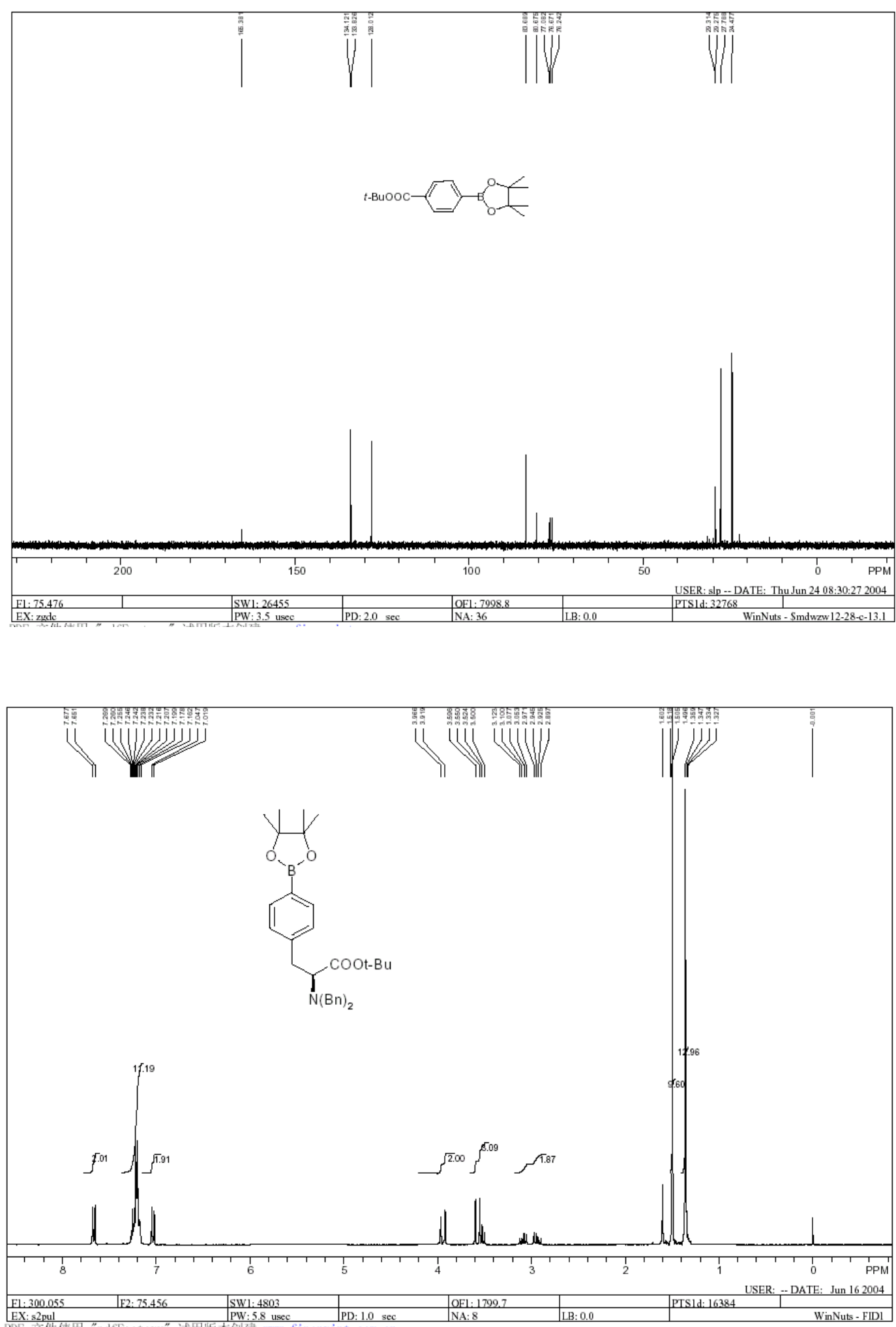


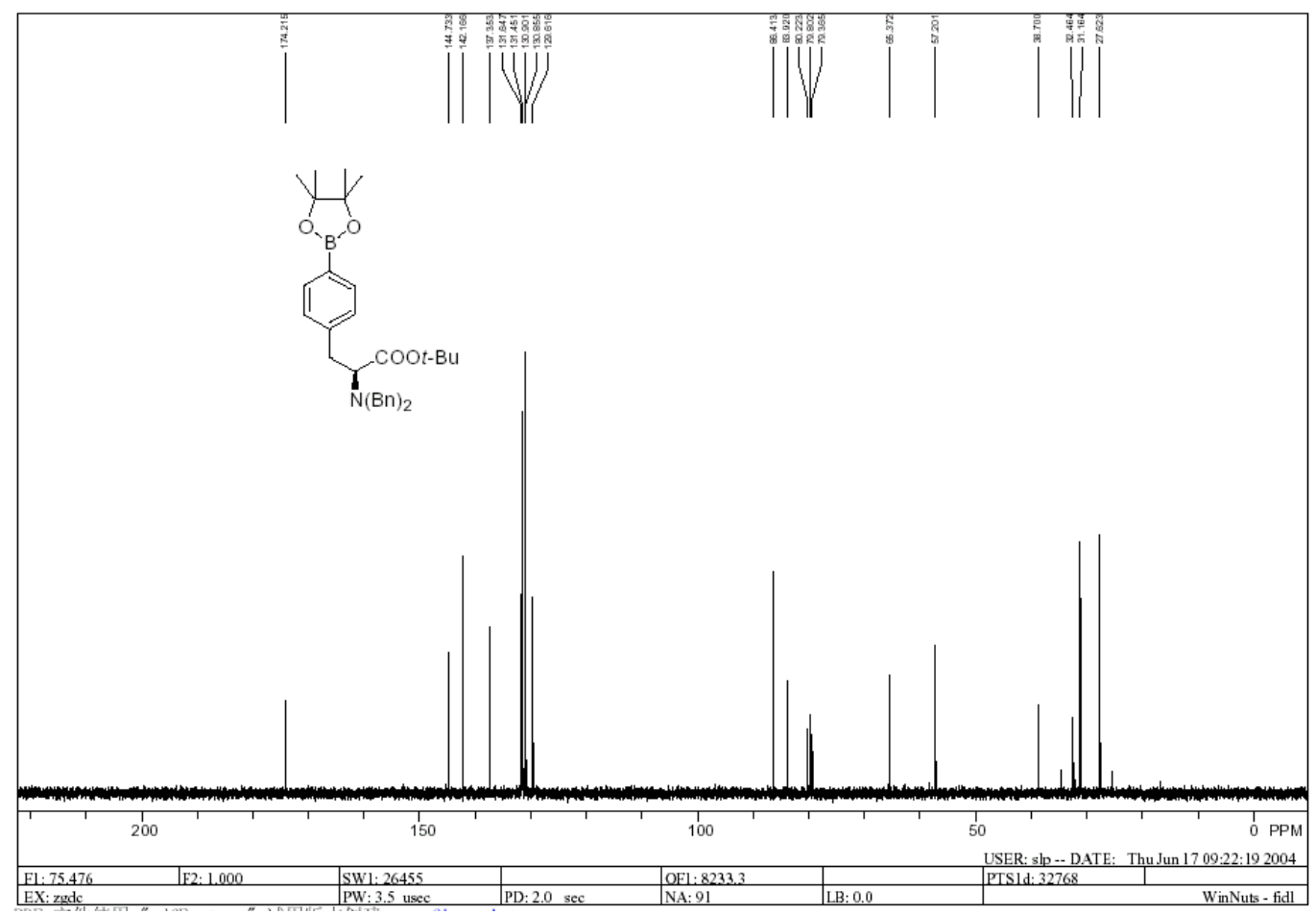

